\title{
Correction to: Gender difference in acquired seizure susceptibility in adult rats after early complex febrile seizures
}

\author{
Yun-Jian Dai ${ }^{1} \cdot{\text { Zheng-Hao Xu }{ }^{1} \cdot \text { Bo Feng }^{1} \cdot \text { Ceng-Lin Xu }^{1} \cdot \text { Hua-Wei Zhao }}^{1} \cdot$ \\ Deng-Chang $\mathrm{Wu}^{1,2} \cdot$ Wei-Wei Hu${ }^{1} \cdot$ Zhong Chen ${ }^{1}$
}

Published online: 28 November 2017

(c) Shanghai Institutes for Biological Sciences, CAS and Springer Nature Singapore Pte Ltd. 2017

Correction to: Neurosci Bull 30(6): 913-922

https://doi.org/10.1007/s12264-014-1482-8

In the original publication of the article, the representative EEG of female rat pups with FS in Figure $1 \mathrm{C}$ and D was incorrectly intercepted from that of male rat pups. This correction does not affect the conclusions of the paper. Figure 1 has been corrected on the online PDF version and displayed below.

The online version of the original article can be found under https:// doi.org/10.1007/s12264-014-1482-8.

Wei-Wei Hu

huww@zju.edu.cn

$\triangle$ Zhong Chen

chenzhong@zju.edu.cn

1 Department of Pharmacology, Key Laboratory of Medical Neurobiology of the Ministry of Health of China, Zhejiang Province Key Laboratory of Neurobiology, Basic Medical College, College of Pharmaceutical Sciences, Zhejiang University, Hangzhou 310058, China

2 Department of Neurology, Collaborative Innovation Center for Diagnosis and Treatment of Infectious Diseases, First Affiliated Hospital, School of Medicine, Zhejiang University, Hangzhou 310003, China 
Fig. 1 No gender difference in the generation of febrile seizures in rat pups. A The latencies to the generation of FSs did not significantly differ in male and female pups ( $n=8$ for both groups). B Male pups had threshold temperatures similar to female pups for the onset of FSs ( $n=8$ for both groups). C, D Hippocampal EEG recordings from freely moving male (C) and female (D) pups. Normal discharges before FSs were replaced by epileptiform discharges after FSs. A nonparametric test was used for the analysis of latency to and threshold of FS generation.
A

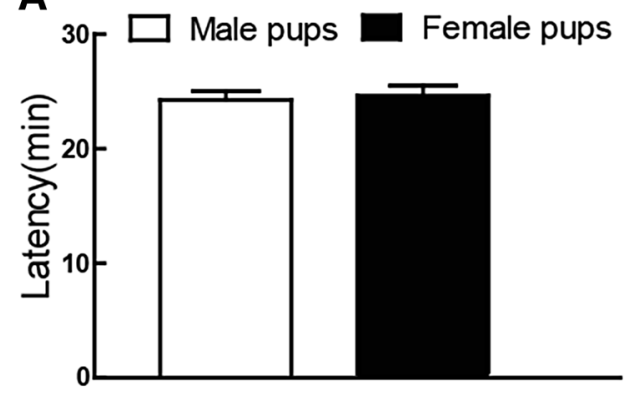

B

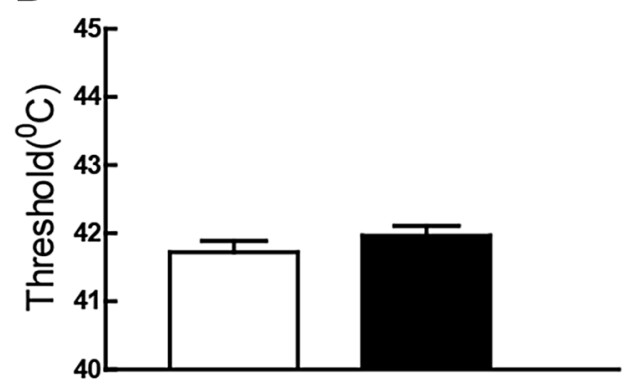

C

Febrile seizure(Male)

D

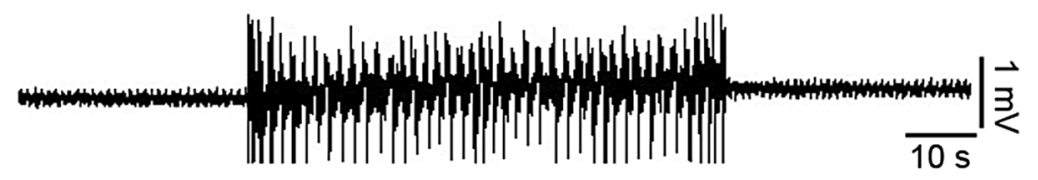

Febrile seizure(Female)

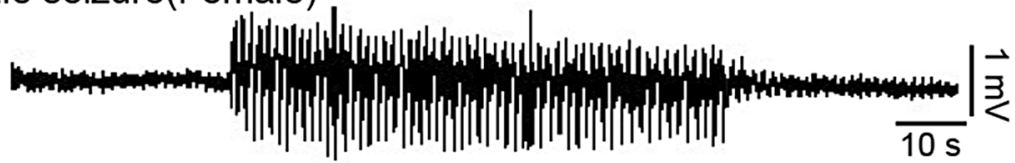

